\title{
Electrochemical Assessments of Droplet Contents in Microfluidic Channels. Application to the Titration of Heterogeneous Droplets.
}

\author{
Thomas Delahaye, Teo Lombardo, Catherine Sella, Laurent Thouin* \\ PASTEUR, Département de chimie, Ecole normale supérieure, PSL University, Sorbonne Université,
} CNRS, 75005, Paris, France

* Corresponding author:

laurent.thouin@ens.psl.eu

\begin{abstract}
Series of aqueous droplets containing redox species were generated on-demand in a microfluidic channel and detected downstream by an electrochemical cell. Depending on the cell geometry, amperometric detections were performed to simultaneously determine the velocity, volume and content of circulating droplets in oil. Volumes and velocities were estimated from specific transition times on the chronoamperometric responses, while charge were evaluated from current integration. The results showed that the total charge within droplets was controlled by the geometry of the electrochemical cell and droplet velocity, leading to accurate determinations of droplet content under specific operating conditions. An active merging of droplets with titrating solutions was tested for analytical purposes. The results demonstrated that even if the mixing was not complete during detection, the assessment of droplet content was still valid. The performance of electrochemical detection was thus evidenced to determine the content of heterogeneous droplets. This property is pertinent in droplet microfluidics since the design of sophisticated circuits is no longer required to fully homogenize the droplet content before characterization, opening broader perspectives in droplet-based microfluidics.
\end{abstract}

Keywords: droplet-based microfluidics; electrochemical detection; titration; dilution curve; microchannel electrode; amperometry. 


\section{INTRODUCTION}

The field of microfluidics emerged in past decades for precise manipulations of fluid samples. In this context, droplet-based microfluidics became an interesting approach for performing highthroughput analysis and screening with very small volumes and flexible control of composition [1-3]. It has undergone great developments and applications in the field of chemistry, biology and materials generation [4]. The benefits rely on the compartmentalization of chemical and biological reactions in droplets used as individual microreactors. On the one hand, reaction of sample with reagents is facilitated improving kinetics and chemical equilibrium. On the other hand, Taylor-Aris diffusion is minimal preventing dilution of analytes for long-term monitoring. Droplet manipulations (i.e., generation, merging, splitting and sorting) enable multistep reactions and complex operations in parallel [5]. Since its introduction, droplet-based microfluidics has now matured in powerful technology set. It integrated large numbers of functional components to automate process and to ensure fine chemical and biological information [6-8]. Maximizing the amount of data that can be extracted from each individual droplet continues to be a relevant challenge. Despite the powerful detection methods used today, analytical detection techniques are still needed to enlarge the application field of droplet-based microfluidics [9]. Indeed, physical and chemical properties of droplets need both to be characterized to deliver highly significant and reliable information. Optical detections based on absorbance and fluorescence detection schemes are the most widely used. They offer highly selective, sensitive and accurate quantifications, but they require good light transmission properties of the optofluidic systems and extended optical pathlengths for high throughput detections [10]. Their main drawback is the instrumentation cost and inadequacy with microsystems for on-site applications due to bulky instrumentations. Alternative droplet detection techniques rely on electrical signals [9]. They provide unique advantages for droplet detection, including the label-free nature, high sensitivity, rapid responses, and miniaturization. Beside impedance-based [11] and capacitance-based [12] detections, electrochemical detection is particularly promising for real time characterization of droplets. Electroactive species can be detected in the flow carrier for high-throughput detection of droplets [1314]. Electroactive species can be also selectively probed inside droplets for analytical determinations 
[15-21]. In this latter case, the major challenge lies in establishing direct contact of the water-in-oil droplets with electrodes while preventing interference of the oil phase. Direct droplet-electrode contacts were achieved by inserting wire electrodes into the center region of a microchannel [15-18]. However, when penetration of electrodes in droplets is forced, droplet instabilities occur leading to lower repeatability of the electrochemical responses. Hence, strategies based on elongation of droplets in narrower section of microchannels were suggested to overcome these drawbacks [22-23]. Phase separation was also proposed by switching the droplet flow by a two-phase laminar flow with treated hydrophobic walls of microchannel [24]. The simplest approach remains the design of electrodes on microchannel walls, with proper sizes to promote the breaking of the surrounding oil film. To ensure efficient contact time with the electrodes, we demonstrated recently that accurate amperometric responses were obtained with droplets having large-aspect ratio [25-26]. In this case, mass transport to electrodes is mainly assured by the internal recirculating convection within droplets with a velocity profile similar to the one of continuous flow [25]. We also showed that some electrochemical cell configurations were more suitable than others due to better cell stability and better performance during the passage of droplets [26]. On the one hand, the velocity and size of droplets can be evaluated from several specific transition times observed on the chronoamperometric curves. On the other hand, the local concentrations in redox species within droplets can be easily assessed from limiting currents. Thus, by using appropriate cell configurations, reliable and self-consistent measurements can be achieved to fully characterize each droplet detected. Note that this approach is relevant since manipulation of droplets often needs a precise control of their properties, which are sensitive to the changes in flow conditions due to discontinuous modulations of pressure during droplet operations. During a given process, both physical and chemical parameters are required to deliver reliable information. Here, the evaluation of the droplet content from chronoamperometric curves relies on the determination of the droplet size and on the local concentrations of electroactive species inside. In this work, we wanted to extend an approach based on a dual-working-electrode configuration (4E) in the case of droplet titration by initiating upstream the active merging of the droplets with titrating solutions. In particular, the objective was to evidence the performance that could be achieved in a short time without the need of a complete homogenization of the droplet content before detection. Indeed, the mixing within droplets 
remains challenging in microfluidics as it is only controlled by molecular diffusion due to the flow stratification of internal recirculation [4-5,27]. In the case of droplets with high aspect ratio, complete homogenization requires time, which at first glance prevents any immediate determinations of their overall content. In the following, electrochemical titrations were assessed within a microfluidic channel whatever the degree of heterogeneity or homogeneity of droplet content reached after active merging.

\section{EXPERIMENTAL}

All devices consisted of classical hybrid glass chips with polydimethylsiloxane (PDMS) blocks (Figure S1). The microfabrication procedure as well as the experimental setup were reported previously [25]. The microchannel network was engraved in the PDMS block while platinum electrodes were patterned on glass by soft-lithography and lift-off procedures. The glass slide was bonded irreversibly with the PDMS block by plasma treatment.

Two designs of microdevice were considered consisting of a same microfluidic network with two different electrochemical cell configurations (Figure 1). The microfluidic network comprises upstream a flow-focusing geometry for on-demand droplet generation, a T-junction for merging an additional volume with the generated droplets, and downstream a linear microchannel that incorporates the electrochemical cell. The distances between the flow-focusing geometry and the T-junction was $6.8 \mathrm{~mm}$ while the distance between the T-junction and the electrochemical cell was $10 \mathrm{~mm}$. The cell configuration 4E (i.e., four electrodes, Figure 1A) was a sequential arrangement of microband platinum electrodes placed perpendicular to the main microchannel with two working electrodes WE1 and WE2, a pseudo-reference electrode RE and a counter electrode CE. A second configuration 5E (i.e., five electrodes, Figure 1B) was also investigated. The only difference with the configuration 4E is the insertion of a large working electrode WE3 between WE2 and RE in order to increase the total active surface of the cell. Higher active surface in the configuration 5E leads to probing a higher fraction of the droplet content under the same experimental conditions compared to the configuration 4E. In both configurations, all electrodes have regular shape, except CE, which has a special geometry to facilitate the detachment of droplet from the electrochemical cell (Figure S2C). All the geometric characteristics 
are reported in Table 1. The width and height of the main microchannel were $200 \mu \mathrm{m}$ and $40 \mu \mathrm{m}$ respectively.

A silicon oil 150 AP Wacker (Sigma-Aldrich) was used as continuous organic phase in the central inlet, while aqueous solutions were used as the dispersed phase in inlets 1 and 2 with $0.5 \mathrm{~mol} \mathrm{~L}^{-1}$ potassium chloride ( $\mathrm{KCl}$, Acros Organics) as the supporting electrolyte. At inlet 1, potassium ferrocyanide $\left(\mathrm{K}_{4} \mathrm{Fe}(\mathrm{CN})_{6}\right.$, Acros Organics) was added in $\mathrm{KCl}$ solutions with concentrations ranging between $210^{-4}$ and $210^{-3} \mathrm{~mol} \mathrm{~L}^{-1}$. At inlet 2, concentration of potassium ferrocyanide was either 0 or $9.810^{-4} \mathrm{~mol} \mathrm{~L}^{-1}$ depending on the type of experiment. The diffusion coefficient $D$ of ferrocyanide anion was equal to $610^{-6} \mathrm{~cm}^{2} \mathrm{~s}^{-1}$ [28]. A pressure controller (MFCS-FLEX, Fluigent) controlled the injections of oil and aqueous solutions in all inlets. Each droplet was generated on demand by applying a pressure pulse over the aqueous solution in inlet 1 . Similarly, for titration by standard addition, the merging of the droplet at the T-junction with a titrant solution of potassium ferrocyanide was performed by applying on demand a pressure pulse over the aqueous solution in inlet 2. Hence, according to the pressure balance in the main channel, droplet velocity was about $500 \mu \mathrm{m} \mathrm{s}^{-1}$, while droplet volumes ranged between 20 and $62 \mathrm{~nL}$.

To optically control the generation and evolution of the droplets along the main microchannel, the microdevices were placed under an inverted microscope (Axiovert 135, Zeiss) equipped with a CCD camera (AxioCam Icc1, Zeiss) (Figure S1A). Photographs of droplets are reported in Figure S2 during generation, active merging and electrochemical detection. When implementing active merging, the volume of the initial droplet generated by flow focusing was assessed optically before its passage through the T-junction. Otherwise, its volume was determined from electrochemical measurements.

Chronoamperometric measurements were carried out using a potentiostat (Autolab type PGSTAT30, Metrohm) associated with a BIPOT module and driven by NOVA software. Two current responses were recorded simultaneously: WE1 and WE2 responses in configuration 4E and WE1+WE2 (connected together) and WE3 in configuration 5E. The working electrodes were biased at $0.4 \mathrm{~V} / \mathrm{RE}$ on the oxidation plateau of the ferrocyanide anion. Before each measurement, the electrodes were activated by performing in the aqueous phase several cyclic potential scans from -0.8 to $1.1 \mathrm{~V} / \mathrm{RE}$, at a potential scan rate of $0.7 \mathrm{Vs}^{-1}$. 
Numerical simulations were performed in a 2D space using COMSOL Multiphysics software. Since the droplets had plug shape with high aspect ratio, the formulation of the problem was identical to those described in previous works considering a continuous flow of aqueous phase [29-32]. Assuming that the electrochemical reaction was only limited by mass transport, the diffusion-convection equation was solved numerically by finite elements according to the cell geometry. As for laminar flow, a parabolic flow profile was considered across the microchannel section with appropriate boundary conditions (Figure S3).

\section{RESULTS AND DISCUSSION}

The determinations presented below rely on the following conditions: (i) The detected droplet contains an aqueous solution of a redox couple that can be electrochemically oxidized or reduced on platinum electrodes; (ii) The working electrodes are biased at a constant potential so that the electrochemical reaction at electrode surfaces is fully controlled by mass transfer, i.e., by diffusion convection.

\subsection{Principle}

The design of the two microfluidic devices is described in Figure 1. Each device comprises one inlet for the carrying fluid (oil), two inlets for the introduction of droplet content (aqueous phases 1 and 2) and one outlet. A flow focusing geometry is used upstream to generate on-demand the former droplet. A Tjunction is located along the main channel in order to inject a given volume of the second aqueous phase within the droplet through active merging. The injection is synchronized with the passage of the droplet over the T-junction and the resulting droplet is detected downstream by the electrochemical cell. Both configurations $4 \mathrm{E}$ and $5 \mathrm{E}$ allow simultaneously the determinations of the final velocity, volume and content of the droplet. On the one hand, as described in a previous work [26], droplet velocity and volume are evaluated based on time transitions monitored during chronoamperometry (Figure 2). On the other hand, the droplet content is estimated by integrating the current responses over time. 
Velocity and volume determinations. Four transition times are considered to determine droplets velocity and volume (Figure 2). $t_{0}$ corresponds to the time when the droplet wets all electrodes, in particular when it contacts CE. $t_{1}$ and $t_{2}$ correspond to the times when the tail end of the droplet leaves WE1 and WE2 respectively. $t_{3}$ is the time in configuration 5E when the droplet leaves WE3.

In both configurations, all the transitions observed in chronoamperometry allow calculating the mean velocity $v$ of the droplet during the time interval $\left(t_{2}-t_{1}\right)$ by:

$$
v=\frac{g_{\mathrm{WE} 1-\mathrm{WE} 2}}{t_{2}-t_{1}}
$$

where $g_{\text {WE1-WE2 }}$ is the gap distance between WE1 and WE2. In addition, the volume $V$ of the droplet is determined by considering the time interval $\left(t_{2}-t_{0}\right)$ given by:

$$
V=h L\left[v\left(t_{2}-t_{0}\right)+g_{\mathrm{WE} 2 \mathrm{CE}}\right]
$$

where $g_{\text {WE2-CE }}$ is the gap distance between WE2 and CE while $h$ and $L$ are the height and width of the main microchannel respectively. Note that Equation (2) can be written considering also the time interval $\left(t_{1}-t_{0}\right)$ and the gap distance $g_{\text {WE1-CE }}$ such as:

$$
V=h L\left[v\left(t_{1}-t_{0}\right)+g_{\mathrm{WE1}-\mathrm{CE}}\right]
$$

Equations (2) and (3) are based on the assumption that droplets are cuboid. It was demonstrated that the curvature at each end does not introduce severe approximations according to the aspect ratios of the droplets, which are rather low in the case of plugs. However, equation (2) will be preferred due to better precision in determining experimentally the corresponding time interval [26]. One example of amperometric responses with configuration $4 \mathrm{E}$ is given in Figure $3 \mathrm{~A}$. In this case, the droplet was generated by flow focusing without performing any injection at the T-junction. The droplet content was homogeneous and the concentration was the one of aqueous phase at inlet 1. As observed in Figure 2, the electrical circuit is established at time $t_{0}$ when the droplet connects all the electrodes. The currents of WE1 and WE2 increase sharply and then decrease to tend to steady-state limiting values controlled by the mass transport of electroactive species within the droplet. WE1 and WE2 are disconnected at times $t_{1}$ and $t_{2}$ as observed by the drop of respective currents to baseline. Note that an additional transition time $t_{\mathrm{d}}$ is noticed on WE2 response between $t_{0}$ and $t_{1}$ corresponding to an electrochemical time 
delay. Indeed, in contrast to the sequences described above, this time is related to a convective coupling between WE1 and WE2. At time $t_{\mathrm{d}}$ the concentration gradient generated by WE1 reaches WE2, leading locally to a depletion of electroactive species, and therefore to a slight decrease of WE2 current. We demonstrated previously that the time interval $\left(t_{\mathrm{d}}-t_{0}\right)$ was inversely proportional to the average velocity of droplet [26]. At times lower than $t_{\mathrm{d}}$, WE1 and WE2 currents are similar since each electrode operates independently without any interaction. All these characteristics of chronoamperometric responses in configuration 4E, including time transitions and current plateaus, were reproduced successfully for each electrode by introducing the droplet velocity $v$ and volume $V$ into the simulations (Figure 3B).

Droplet content determination. Droplet content can be assessed through different approaches by integrating (i) the current of WE1 or WE2 (configuration 4E), (ii) the current of WE1 or WE2 or WE3 (configuration 5E), or (iii) by summing the currents of all working electrodes (configurations $4 \mathrm{E}$ and 5E). However, despite the approach used for a given cell geometry and configuration, the determination of droplet content depends on the experimental velocity and volume of droplets. Indeed, according to the velocity and thus to the internal recirculating convection within droplets, each working electrode probes only a layer of fluid above its surface. This situation is similar to the known operating regimes of channel electrodes in a continuous flow of aqueous solution (i.e., with no segmented flow) $[29,33-$ 34]. In addition, the volume of droplet probed by each working electrode is determined by their respective position within the electrochemical cell (Figure 2). Therefore, for a given cell geometry, calibrations are needed to account for the influence of velocity and volume of detected droplets. Moreover, it must be noticed that the length of droplet is not quantitatively probed by the electrochemical cell since a small part of the droplet already passed over the working electrodes at time $t_{0}$ before the operation of the electrochemical cell. This drawback can be minimized when the gap distance between WE2 and CE (configuration 4E) or between WE3 and CE (configuration 5E) is negligible compared to the overall droplet length (see below). 


\subsection{Assessment of electrical charges}

Case of homogeneous droplets. Some preliminary experiments were performed by determining the charge quantities for homogeneous droplets, i.e., without modifying their initial composition by means of the T-junction. Concentration $c$ of the aqueous phase ranged between $210^{-4}$ to $210^{-3} \mathrm{~mol} \mathrm{~L}^{-1}$ while volumes and velocities of droplets were kept almost constant to meet the criteria needed for charge determinations. Using configuration $4 \mathrm{E}$, the charges $Q_{\mathrm{d}}$ were evaluated by integrating the amperometric responses of WE1, WE2 and their sum (Figure 4A). The values were compared with the charge $Q$ estimated from the volume $V$ of each droplet and concentration $c$ (i.e., by $Q=\mathrm{F} c V$ ). Note that $V$ was determined electrochemically from transition times using Equations (1) and (2). By integrating individual currents or their sum, linear variations were observed in Figure 4A demonstrating the relation between charges $Q_{\mathrm{d}}$ and $Q$. Slopes corresponded thus to the experimental detection rates $\Gamma_{\exp }$. Values were estimated by linear regression and compared to the theoretical detection rates $\Gamma_{\text {theo }}$ obtained from simulated current responses (see Figure 3B) taking into account the cell geometry, average volume $V_{\text {av }}$ and average velocity $v_{\mathrm{av}}$ of the series of droplets. As shown in Table 2, a very good agreement was observed between data. In this case, the electrodes probe together up to $24 \%$ of the droplet contents (i.e., value obtained from the slope in Figure 4A) according to the operating regimes established at the electrodes. Indeed, under such experimental conditions, the average flow velocity above the electrode is high enough so that the electrodes cannot probe all the quantity of species passing above their surface but only a fraction [29]. The difference in detection rates between WE1 and WE2 is small but indicative of the convective coupling between the two electrodes. Similar results were derived from Figure 4B by comparing the detected charge $Q_{\mathrm{d}}$ with the concentration $c$ within droplets. All the results show that within the experimental uncertainties, data were consistent with theory, validating the whole procedure based on current integration with in-situ determinations of $V$ and $v$ by transition times. Droplet contents can be quantitatively determined if the detection rate is estimated theoretically or established experimentally as in Figure 4A.

Case of heterogeneous droplets. Similar experiments were conducted after active merging of droplets in the microfluidic circuit: a droplet of volume $V_{1}$ is generated by the flow focusing geometry and a 
volume $V_{2}$ of a second aqueous phase is added at the T-junction. In the following, the concentration of the aqueous phase at inlet 1 was $c_{1}=210^{-3} \mathrm{~mol} \mathrm{~L}^{-1}$ whereas the concentration of the aqueous phase at inlet 2 (T-junction) was $c_{2}=0$. Under these conditions, the droplet content was set according to the respective volumes $V_{1}$ and $V_{2}$. Some examples of amperometric responses are reported in Figures $3 \mathrm{C}$ and $3 \mathrm{E}$ for configurations $4 \mathrm{E}$ and $5 \mathrm{E}$ respectively. In comparison to homogeneous droplets (Figure $3 \mathrm{~A}$ ), currents increase also sharply at time $t_{0}$ but fluctuate after without reaching any steady-state plateaus during droplet detection: This divergence was confirmed by simulating the current responses under the same operating conditions, assuming that the droplet contents were homogeneous upon detection (Figures 3D and 3F). The differences in Figures 3C-E between experimental and simulated variations were thus indicative of the droplet heterogeneity. Indeed, the time interval between the addition of aqueous phase and droplet detection was too short for the mixture to homogenize by a simple diffusion process. Compared to homogeneous droplets (Figure 3A), time transitions associated to the connection - disconnection of working electrodes are also clearly observed (Figures 3C and 3E), except for the electrochemical time delay $t_{\mathrm{d}}$. In configuration $4 \mathrm{E}$, the current variation resulting from droplet heterogeneity hides the slight deviation expected at $t_{\mathrm{d}}$ (compare Figures $3 \mathrm{C}$ and $3 \mathrm{D}$ ). As the active merging dilutes the droplets in their middle, a decrease followed by an increase in current is observed for each electrode before disconnection. Note that the experimental responses were not reproducible under same experimental conditions since the active merging during the passage of droplet was triggered manually.

Determinations of charge $Q_{\mathrm{d}}$ were then performed in both configurations for series of droplets having similar volumes $V$ and velocities $v$. Data are reported in Figure 5 in configuration $5 \mathrm{E}$ for different sizes of WE3. Like for homogeneous droplets (Figure 4A), $Q_{\mathrm{d}}$ was plotted as a function of $Q_{1}$ with $Q_{1}=\mathrm{F} c_{1} V_{1}$. $Q_{\mathrm{d}}$ was evaluated by integrating the sum of the amperometric responses WE1+WE2 and WE1+WE2+WE3 (see experimental section). Note here that $V_{1}$ was evaluated optically, upstream of the T-junction, whereas $V$ was determined from the transition times using Equations (1) and (2). Despite the heterogeneity within droplets due to weak mixing between aqueous phases, linear variations were still observed. In addition, the slopes increased with the electrode width $w_{\mathrm{WE}}$. At first glance, this overall 
situation was unexpected since the amperometric responses in Figure 3E contrasted significantly with those in Figure 3A. These results showed that the detected charge $Q_{\mathrm{d}}$ remains proportional to the charge within droplet, whether the content is homogeneous or not. They were confirmed by comparing in Table 2 the experimental detection rates $\Gamma_{\exp }$ evaluated from linear regressions (Figure 5) with theoretical ones $\Gamma_{\text {theo }}$ simulated in the case of homogeneous droplets. As previously, a good agreement was observed between data validating the simulations based on the operating regimes of electrode. Under these conditions, the detection rate is only a function of the cell geometry and droplet velocity. Since the electrodes probe thin layers of solution in their proximity, the droplet content is necessarily homogeneous over the droplet height and heterogeneous along the droplet length during electrochemical detection. This is due to the diffusion length of species within droplets in the time interval between injection and detection. During this transition, droplets can fully re-homogenize over their height while partially over their length. Indeed, by considering a velocity $v=500 \mu \mathrm{m} \mathrm{s}^{-1}$, the time interval between the T-junction and electrochemical cell is about $20 \mathrm{~s}$. In comparison, the time required for the droplet content to homogenize over the height $h$ is lower and estimated to $h^{2} / D \sim 3 \mathrm{~s}$. On the contrary, the time required for a droplet of volume $V=32 \mathrm{~nL}$ to homogenize over its length is much higher and equal to $(V / h l)^{2} / D \sim 300 \mathrm{~s}$. Therefore, if the titration was limited to the detection of homogeneous droplets, it would require more time. Moreover, considering a velocity $v=500 \mu \mathrm{m} \mathrm{s}^{-1}$ and a time interval of $300 \mathrm{~s}$, the distance travelled by the droplet is approximately $15 \mathrm{~cm}$, which could limit the design of the most sophisticated microfluidic circuits.

Figure $6 \mathrm{~A}$ reports the detected charges $Q_{\mathrm{d}}$ for heterogeneous droplets in configuration $4 \mathrm{E}$. In contrast to Figure 5, series of experiments were performed with different volumes $V$ from a series to another. Under these conditions, linear variations were still observed in Figure 6A leading to the same conclusions. As expected, $V$ does not influence the operating regimes of electrodes. However, it controls the detection rate as shown in Figure $6 \mathrm{~B}$ by plotting $\Gamma_{\text {theo }}$ and $\Gamma_{\text {exp }}$ as a function of $V$. Indeed, depending on the droplet length (or volume $V$ ), the contribution of the non-probed zone is relative. In configuration $4 \mathrm{E}$, it is the distance between WE2 and CE which defines at time $t_{0}$ the non-probed zone and then the minimal volume $V_{\min }$ of droplet required for performing such determinations. Therefore, for a given cell geometry 
with $V$ greater than $V_{\min }, \Gamma$ increases with $V$ and tends to a limiting value which is a function of $v$ (Figure 6B). Note that the error associated with the charge underestimation decreases by increasing $V$.

The degree of droplet heterogeneity was assessed experimentally from the response of WE2 (Figure 3C) by comparing the current standard deviation $\sigma$ and the average current $i_{\text {av }}$ of WE2 by:

$$
\frac{\sigma}{i_{\mathrm{av}}}=\sqrt{\frac{\sum_{i=1}^{N}\left(i_{\mathrm{i}}-i_{\mathrm{av}}\right)^{2}}{N i_{\mathrm{av}}}}
$$

When the droplet is homogeneous as in Figure 3A, current plateaus are expected and $\sigma / i_{\text {av }}$ tends to zero. In contrast, when the droplet is heterogeneous as in Figure $3 \mathrm{C}, \sigma / i_{\text {av }}$ is higher than zero and is set by the mixing efficiency achieved upon detection between $V_{1}$ and $V_{2}$. Values of $\sigma / i_{\text {av }}$ were thus calculated from data in Figure 6A and plotted in Figure $6 \mathrm{C}$ as a function of the volume ratio $V_{2} /\left(V_{1}+V_{2}\right)$. For each series of droplets, $\sigma / i_{\text {av }}$ tends within the experimental errors towards a maximum for $V_{2} /\left(V_{1}+V_{2}\right)=0.5$. This behavior was predicted theoretically. Indeed, by considering Equation (4), a theoretical profile similar to the experimental variations was established assuming no mixing between $V_{1}$ and $V_{2}$. This profile is reported in Figure $6 \mathrm{C}$ (dotted line) and relies on maximal values of $\sigma / i_{\mathrm{av}}$. Mixing efficiencies were estimated for each series of droplets by fitting this profile with the experimental variations of $\sigma / i_{\text {av. }}$ In this case, mixing efficiencies correspond to proportionality coefficients introduced for the fits (Figure 6C). Hence, despite the fact that experiments were not necessarily reproducible when adding $V_{2}$, the variations correlate well with the droplet volumes. Indeed, in Figure $6 \mathrm{C}$ lower volumes $V$ lead to less heterogeneous droplet contents, due to shorter times required for re-homogenization by diffusion.

\subsection{Application to quantitative determination of droplet contents}

Calibrations curves. The results above demonstrated the reliability of the electrochemical detection for determining charges within droplets regardless of their degree of heterogeneity. Quantitative assessments of droplet content can be thus performed provided that the detection rates are preliminary determined. Detection rates can be evaluated experimentally by establishing calibration curves like in Figures $4 \mathrm{~A}$ and 5 or they can be calculated theoretically through appropriate simulations. For a given 
cell geometry, the velocity $v$ and volume $V$ of the droplets must be kept constant in a series of measurements since both determine the detection rate, as demonstrated above.

Titrations. Active merging allows the titration of droplet contents according to this electrochemical strategy. Note that the latter is true only without chemical or redox reactions. Average concentration $c_{\mathrm{d}}$ within droplet can be derived from charge $Q_{\mathrm{d}}$, droplet volume $V$, and detection rate $\Gamma$ by the equation:

$$
c_{\mathrm{d}}=\frac{Q_{\mathrm{d}}}{\Gamma \mathrm{F} V}
$$

With the addition of an extra volume $V_{2}$ of concentration $c_{2}$ to a volume $V_{1}$ of concentration $c_{1}, c_{\mathrm{d}}$ is the experimental determination of the concentration $c$ given by:

$$
c=\frac{c_{1} V_{1}+c_{2} V_{2}}{V_{1}+V_{2}}
$$

Concentration $c$ is theoretical since it corresponds to the concentration that would be reached within droplet only after complete mixing. As demonstrated previously in Figures 5 and 6A, and according to Equation (5), $c_{\mathrm{d}}$ can be derived from amperometric responses with a good accuracy in the case of heterogeneous droplets. Indeed, dilution curves with $c_{2}=0$ were established from data in Figures 5 and 6A. They are reported in Figure 7 by plotting the concentration ratio $c_{d} / c_{d}{ }^{0}$ as a function of the volume ratio $V_{2} /\left(V_{1}+V_{2}\right)$ where $c_{\mathrm{d}}{ }^{0}$ is the detected concentration when $V_{2}=0$. As observed in Figure 7 , the data follow the linear variations predicted for dilution curves with a decrease of $c_{\mathrm{d}} / \mathrm{c}_{\mathrm{d}}{ }^{0}$ from 1 to 0 . Note that these plots are similar to those given in Figures 5 and 6A since they are only based on a new graphical data processing. They demonstrate again that the experimental measurements performed under these conditions are self-consistent for titration purposes. In addition, Figure 7 offers a direct comparison of the results obtained from the configurations $4 \mathrm{E}$ and $5 \mathrm{E}$, showing that even a relatively small active surface (configuration 4E) allows a reliable measurement of the content of heterogeneous droplets. In the following, a titration based on standard addition procedure was carried out by implementing active merging of two aqueous phases containing both the electroactive species at different concentration $c_{1}$ and $c_{2}$ (with $c_{1}>0$ and $c_{2}>0$ ). In this case, the detected charge $Q_{\mathrm{d}}$ for every droplets equals to:

$$
Q_{\mathrm{d}}=\Gamma \mathrm{F}\left(c_{1} V_{1}+c_{2} V_{2}\right)
$$


where $c_{1}$ is the unknown concentration to be determined and $c_{2}$ the concentration of titrant. Equation (7) can be rewritten as the following:

$$
\frac{Q_{\mathrm{d}}}{V_{1}}=\Gamma \mathrm{F}\left(c_{1}+\frac{c_{2} V_{2}}{V_{1}}\right)
$$

Therefore, by plotting $Q_{\mathrm{d}} / V_{1}$ as a function of $c_{2} V_{2} / V_{1}$, a linear variation is expected experimentally with an intercept on $\mathrm{X}$-axis equal to $-c_{1}$. The determination of $c_{1}$ relies on both the control and in-situ determination of volumes $V_{1}$ and $V_{2}$. As a result, concentration of the titrant $c_{2}$ does not need to greatly exceed concentration $c_{1}$ as in usual procedures of standard addition. One example of titration is reported in Figure 8 when $c_{2}$ is higher than $c_{1}$. Under this experimental condition, concentration $c_{1}$ was determined accurately within $2 \%$ discrepancy. Thus, without any optimization, the limit of detection LOD was evaluated from the slope and 3 times the standard deviation at $10^{-4} \mathrm{~mol} \mathrm{~L}^{-1}$. Following the same procedure, similar results were obtained for titrations carried out when $c_{2}$ was lower than $c_{1}$. Note that higher performance is expected by, for example, increasing the number of droplets and / or by increasing the droplet volumes in order to span large variations of concentration during titrations.

\section{CONCLUSION}

Electrochemical cells have been investigated in microchannels to determine the volume, velocity and content of droplets with high-aspect ratio. The results showed that the droplet characteristics can be determined electrochemically from the amperometric responses by transition times and electrical charges. In-situ determinations of droplet volume and velocity are both required to estimate the electrochemical detection rate and then the content of electroactive species. The experimental data were in good agreement with theory whatever the degree of droplet heterogeneity after active merging. This result stems from the fact that homogenization by diffusion within droplets is reached on the scale of electrodes but not on the droplet lengths during electrochemical detection. Under these conditions, active merging allows titration of droplets even if mixtures with titrating solutions remain incomplete. This property is relevant in droplet microfluidics since it permits the determination 
of contents without requiring specific microfluidic circuits aiming at fully homogenize the droplets before their characterization.

\section{ACKNOWLEDGMENTS}

This work was supported in parts by CNRS UMR 8640, ENS (Ecole normale superieure), PSL University and Sorbonne Université. T.L. thanks ENS and Collegio Superiore of Bologna for being able to participate in a student exchange program. 


\section{REFERENCES}

[1] H. Song, D. L. Chen, R. F. Ismagilov, Angew. Chem. Int. Ed. 45 (2006) 7336.

[2] S. Y. Teh, R. Lin, L. H. Hung, A. P. Lee, Lab Chip 8 (2008) 198.

[3] A. Huebner, S. Sharma, M. Srisa-Art, F. Hollfelder, J. B. Edel, A. J. Demello, Lab Chip 8 (2008) 1244.

[4] L. Shang, Y. Cheng, Y. Zhao, Chem. Rev. 117 (2017) 7964.

[5] R. Seemann, M. Brinkmann, T. Pfohl, S. Herminghaus, Rep. Prog. Phys. 75 (2012) 016601/1.

[6] J. Sanchez Barea, J. Lee, D. K. Kang, Micromachines (Basel) 10 (2019)

[7] A. Suea-Ngam, P. D. Howes, M. Srisa-Art, A. J. deMello, Chem. Commun. 55 (2019) 9895.

[8] K. Matula, F. Rivello, W. T. S. Huck, Adv. Biosys. 4 (2020) e1900188.

[9] W. W. Liu, Y. Zhu, Anal. Chim. Acta 1113 (2020) 66.

[10] T. Yang, S. Stavrakis, A. deMello, Anal. Chem. 89 (2017) 12880.

[11] M. Marcali, C. Elbuken, Lab Chip 16 (2016) 2494.

[12] P. K. Isgor, M. Marcali, M. Keser, C. Elbuken, Sens. Actuators B-Chem. 210 (2015) 669.

[13] S. Liu, Y. Gu, R. B. Le Roux, S. M. Matthews, D. Bratton, K. Yunus, A. C. Fisher, W. T. Huck, Lab Chip 8 (2008) 1937.

[14] Y. Gu, A. C. Fisher, The Analyst 138 (2013) 4448.

[15] Z. Han, W. Li, Y. Huang, B. Zheng, Anal. Chem. 81 (2009) 5840.

[16] Z. Han, Y. Y. Chang, S. W. Au, B. Zheng, Chem. Commun. 48 (2012) 1601.

[17] S. Gu, Y. Lu, Y. Ding, L. Li, F. Zhang, Q. Wu, Anal. Chim. Acta 796 (2013) 68.

[18] S. Gu, Y. Lu, Y. Ding, L. Li, H. Song, J. Wang, Q. Wu, Biosens. Bioelectron. 55 (2014) 106.

[19] X. Hu, X. Lin, Q. He, H. Chen, J. Electroanal. Chem. 726 (2014) 7.

[20] A. Suea-Ngam, P. Rattanarat, O. Chailapakul, M. Srisa-Art, Anal. Chim. Acta 883 (2015) 45.

[21] P. Rattanarat, A. Suea-Ngam, N. Ruecha, W. Siangproh, C. S. Henry, M. Srisa-Art, O. Chailapakul, Anal. Chim. Acta 925 (2016) 51.

[22] F. Sassa, H. Laghzali, J. Fukuda, H. Suzuki, Anal. Chem. 82 (2010) 8725. 
[23] H. Liu, R. M. Crooks, Lab Chip 13 (2013) 1364.

[24] X. Lin, X. Hu, Z. Bai, Q. He, H. Chen, Y. Yan, Z. Ding, Anal. Chim. Acta 828 (2014) 70.

[25] T. Abadie, C. Sella, L. Thouin, Electrochem. Commun. 80 (2017) 55.

[26] T. Lombardo, L. Lancellotti, C. Souprayen, C. Sella, L. Thouin, Electroanalysis 31 (2019) 2103.

[27] O. Kašpar, A. H. Koyuncu, A. Hubatová-Vacková, M. Balouch, V. Tokárová, RSC Adv. 10 (2020) 15179.

[28] C. Amatore, S. Szunerits, L. Thouin, J.-S. Warkocz, Electrochem. Commun. 2 (2000) 353.

[29] C. Amatore, N. Da Mota, C. Sella, L. Thouin, Anal. Chem. 79 (2007) 8502.

[30] C. Amatore, C. Lemmer, P. Perrodin, C. Sella, L. Thouin, Electrochem. Commun. 13 (2011) 1459.

[31] C. Amatore, C. Lemmer, C. Sella, L. Thouin, Anal. Chem. 83 (2011) 4170.

[32] T. Abadie, C. Sella, P. Perrodin, L. Thouin, Front. Chem. 7 (2019) 704.

[33] C. Amatore, N. Da Mota, C. Lemmer, C. Pebay, C. Sella, L. Thouin, Anal. Chem. 80 (2008) 9483.

[34] C. Amatore, N. Da Mota, C. Sella, L. Thouin, Anal. Chem. 82 (2010) 2434. 


\begin{tabular}{|c|c|c|}
\hline & $\begin{array}{c}\text { Configuration 4E } \\
/ \mu \mathrm{m}\end{array}$ & $\begin{array}{c}\text { Configuration 5E } \\
/ \mu \mathrm{m}\end{array}$ \\
\hline$w$ WE1 & 50 & 50 \\
\hline$g_{\text {WE1-WE2 }}$ & 1000 & 1000 \\
\hline$w_{\text {WE2 }}$ & 50 & 50 \\
\hline$g_{\text {WE2-RE }}$ & 100 & - \\
\hline$g_{\text {WE2-WE3 }}$ & - & 400 \\
\hline$w$ WE3 & - & 100 \\
\hline$g_{\text {WE3-RE }}$ & - & 50 \\
\hline$w_{\text {RE }}$ & 50 & 100 \\
\hline$g_{\text {RE-CE }}$ & 100 & $350,550,850$ \\
\hline$w_{\text {CE }}$ & 150 & \\
\hline
\end{tabular}

Table 1: Geometric characteristics of the electrochemical cells according to configurations 4E and 5E. $w$ is the electrode width and $g$ the gap distance between the electrodes. 


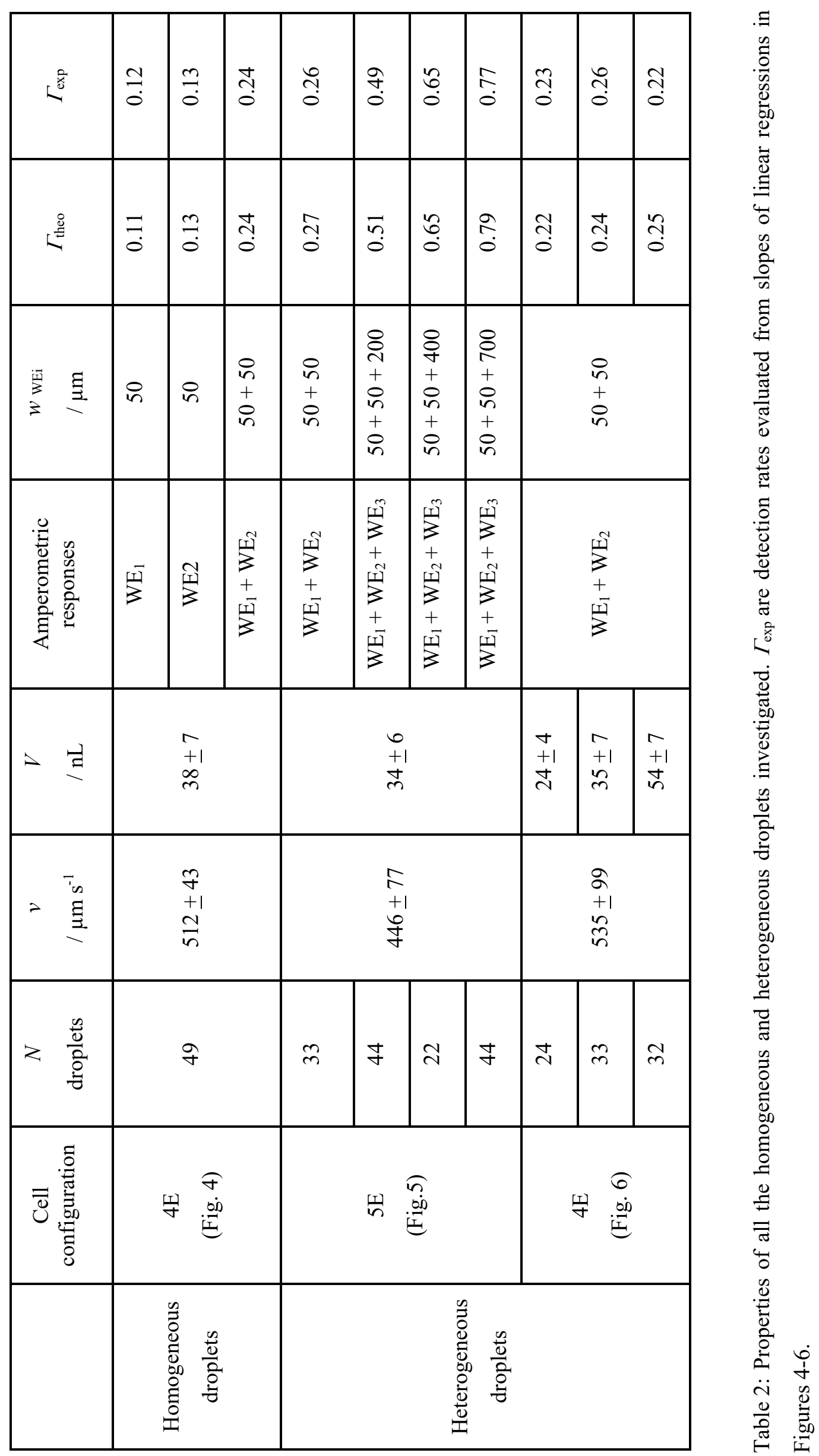




\section{FIGURES}

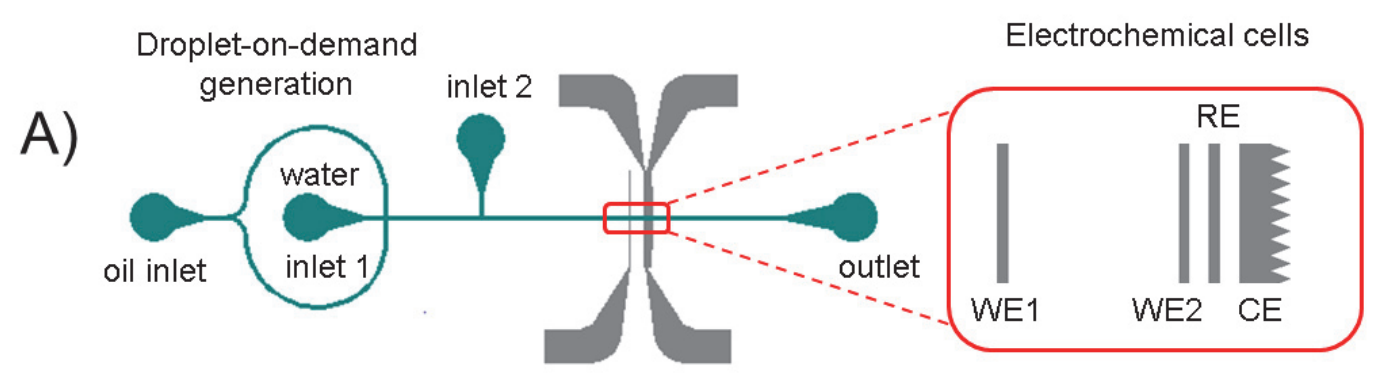

B)

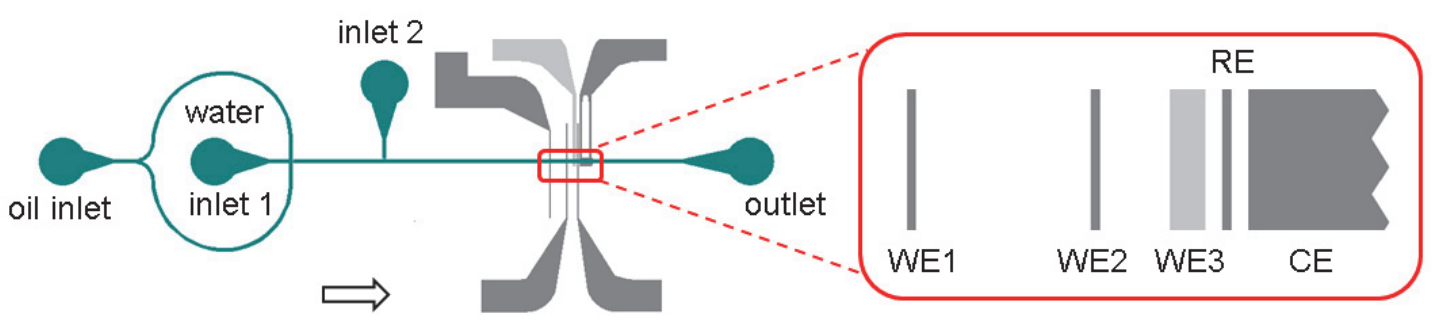

Flow direction

Figure 1: Design of the two microfluidic devices that integrate upstream a flow-focusing geometry for on-demand droplet generation, a T-junction for volume addition, and downstream the electrochemical cell. A) Electrochemical cell configuration 4E with electrodes WE1, WE2, RE and CE. B). Electrochemical cell configuration 5E with electrodes WE1, WE2, WE3, RE and CE. The characteristic dimensions of the electrochemical cells are reported in Table 1. 


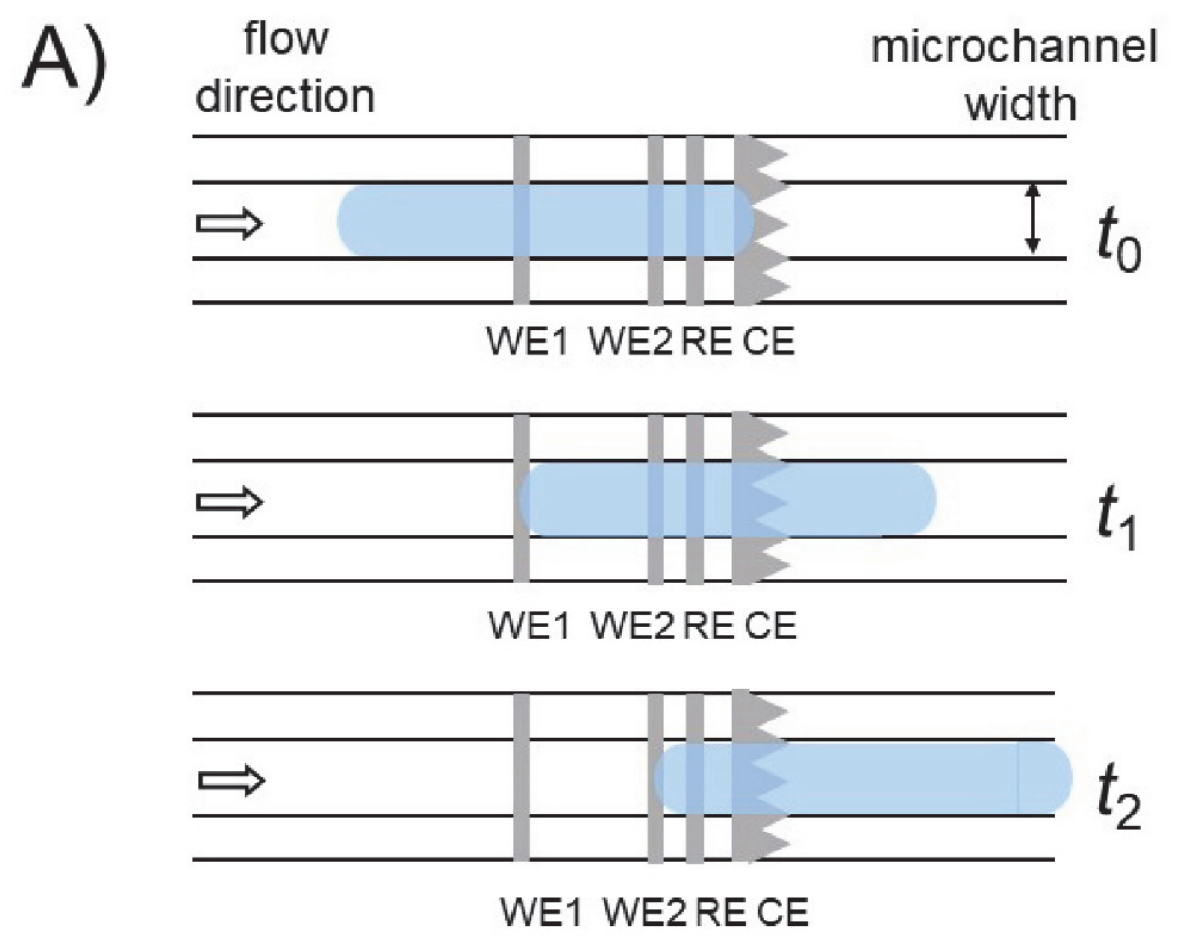

B)

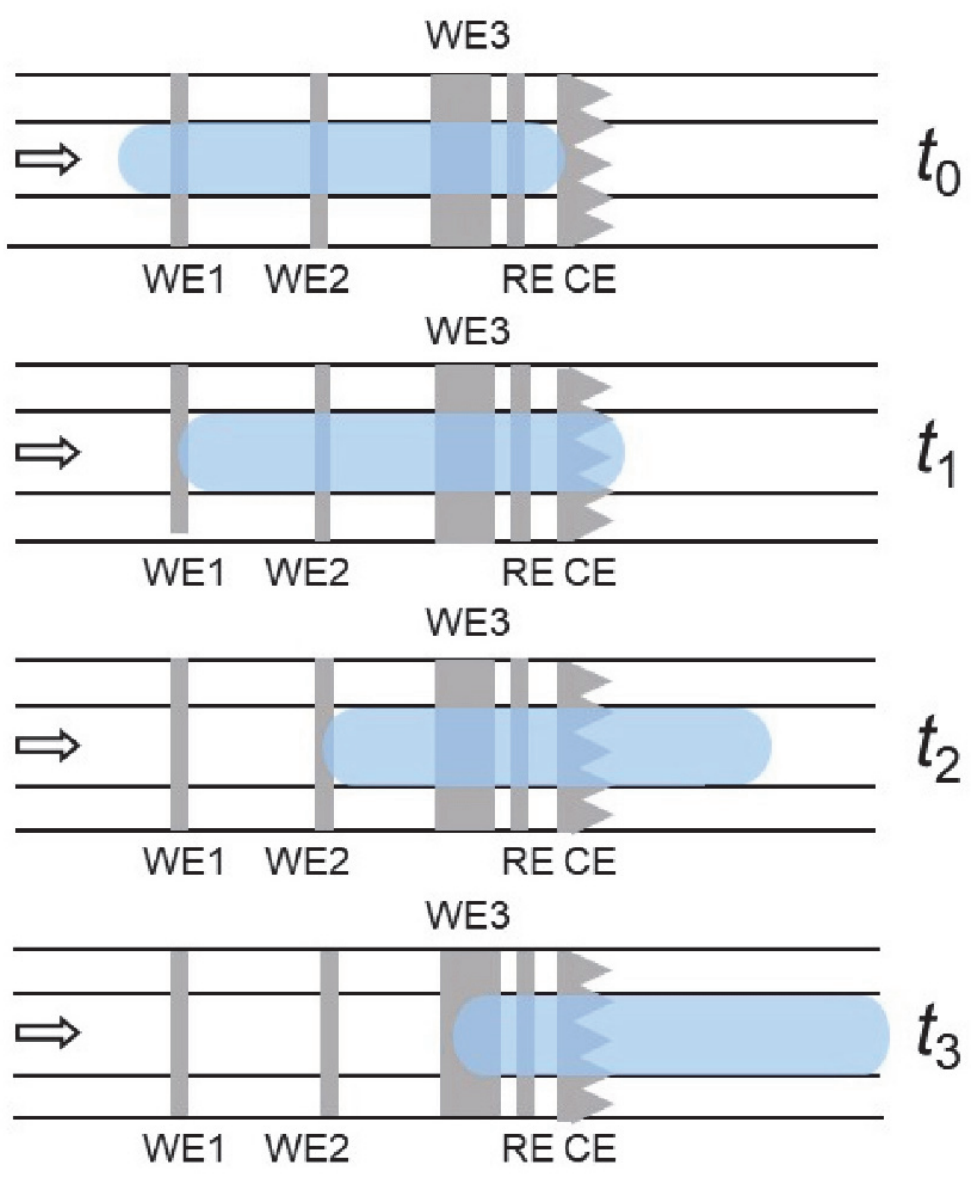

Figure 2: Schematic illustrations for the characteristic times $t_{0}, t_{1}, t_{2}$ and $t_{3}$ during the circulation of the droplet over the electrochemical cell for configurations 4E (A) and 5E (B). 

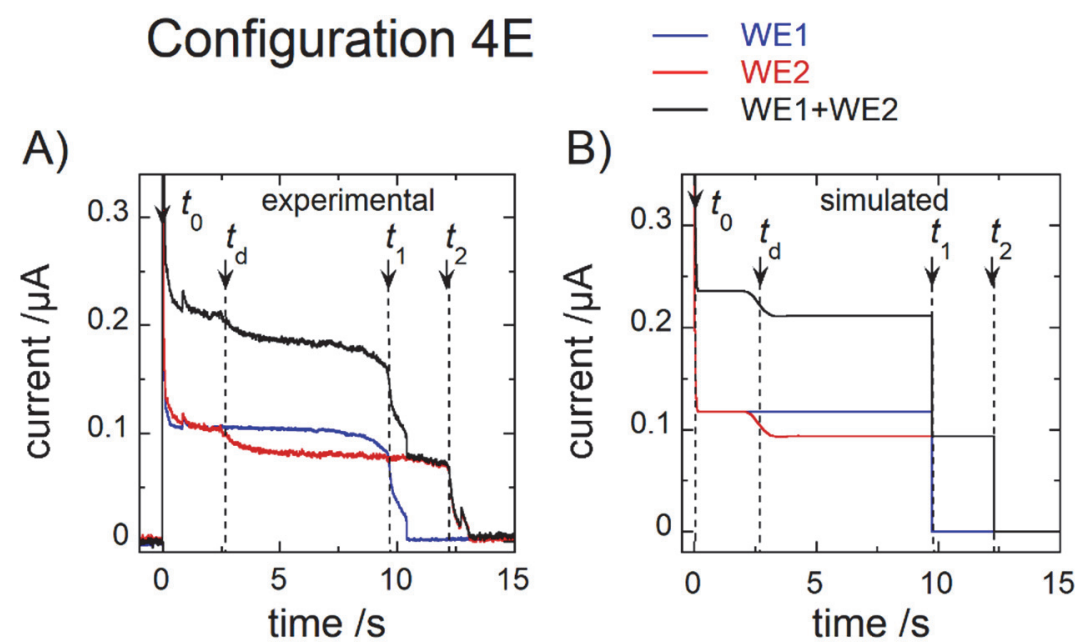

C)
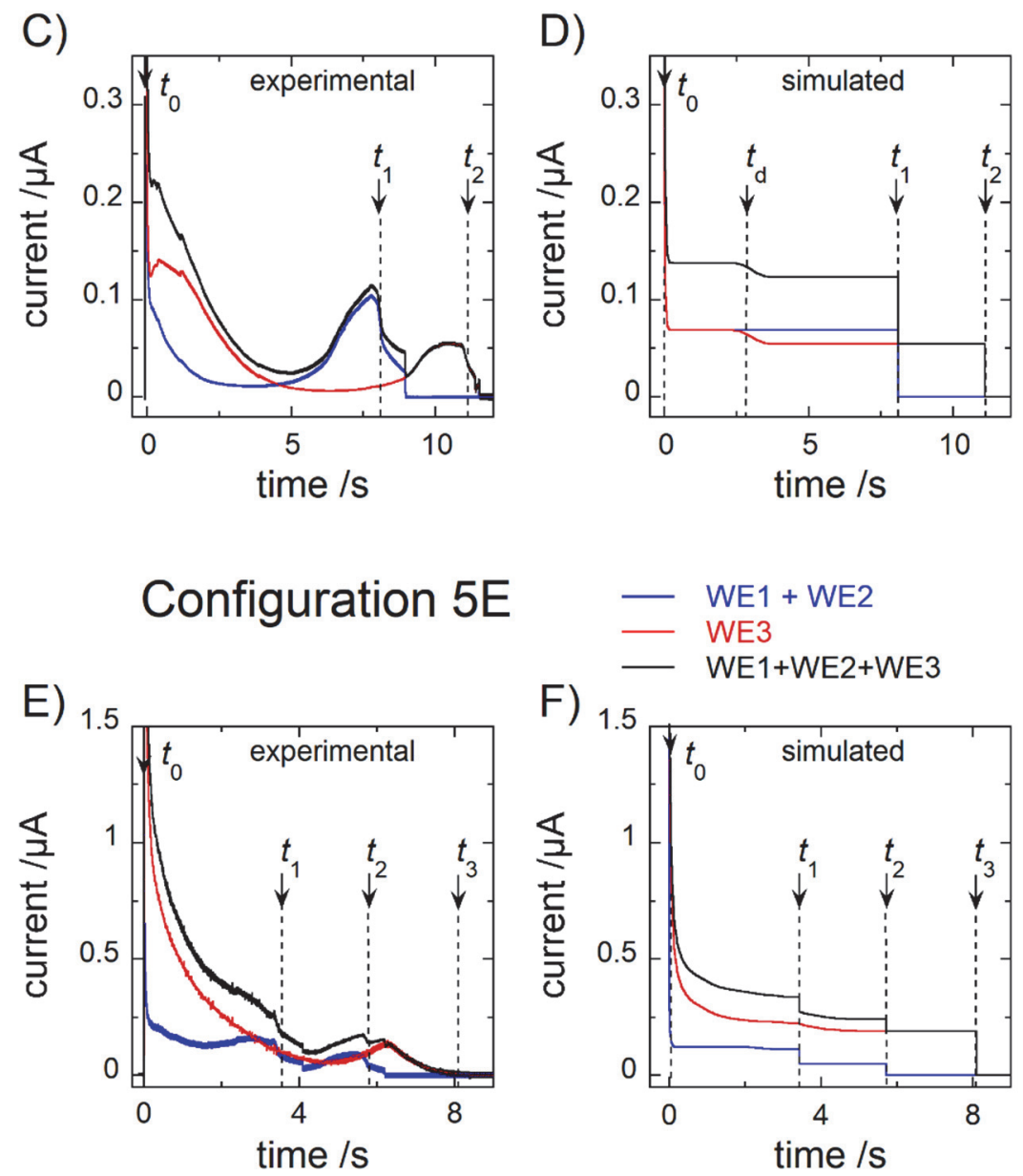

Figure 3: Comparison between experimental and simulated chronoamperometric responses during droplet detection. A-B) Detection of a homogeneous droplet in configuration $4 \mathrm{E}$ with $v=394 \mu \mathrm{m} \mathrm{s}^{-1}$, and $V=41 \mathrm{~nL}$. (C-D) Detection of a heterogeneous droplet in configuration $4 \mathrm{E}$ with $v=333 \mu \mathrm{m} \mathrm{s}^{-1}$, $V_{1}=20 \mathrm{~nL}$, and $V=32 \mathrm{~nL}$. (E-F) Detection of a heterogeneous droplet in configuration E5 with $v=436 \mu \mathrm{m} \mathrm{s}^{-1}, V_{1}=16 \mathrm{~nL}$, and $V=31 \mathrm{~nL}$. In all cases, $w_{\mathrm{WE} 1}=w_{\mathrm{WE} 2}=50 \mu \mathrm{m}, w_{\mathrm{WE} 3}=700 \mu \mathrm{m}$, $E=0.4 \mathrm{~V} / \mathrm{RE}, c=c_{1}=210^{-3} \mathrm{~mol} \mathrm{~L}^{-1}$, and $c_{2}=0 . t_{0}, t_{1}, t_{2}$ and $t_{3}$ correspond to the characteristic times defined in Figure 2. 


\section{Homogeneous droplets - Configuration 4E}
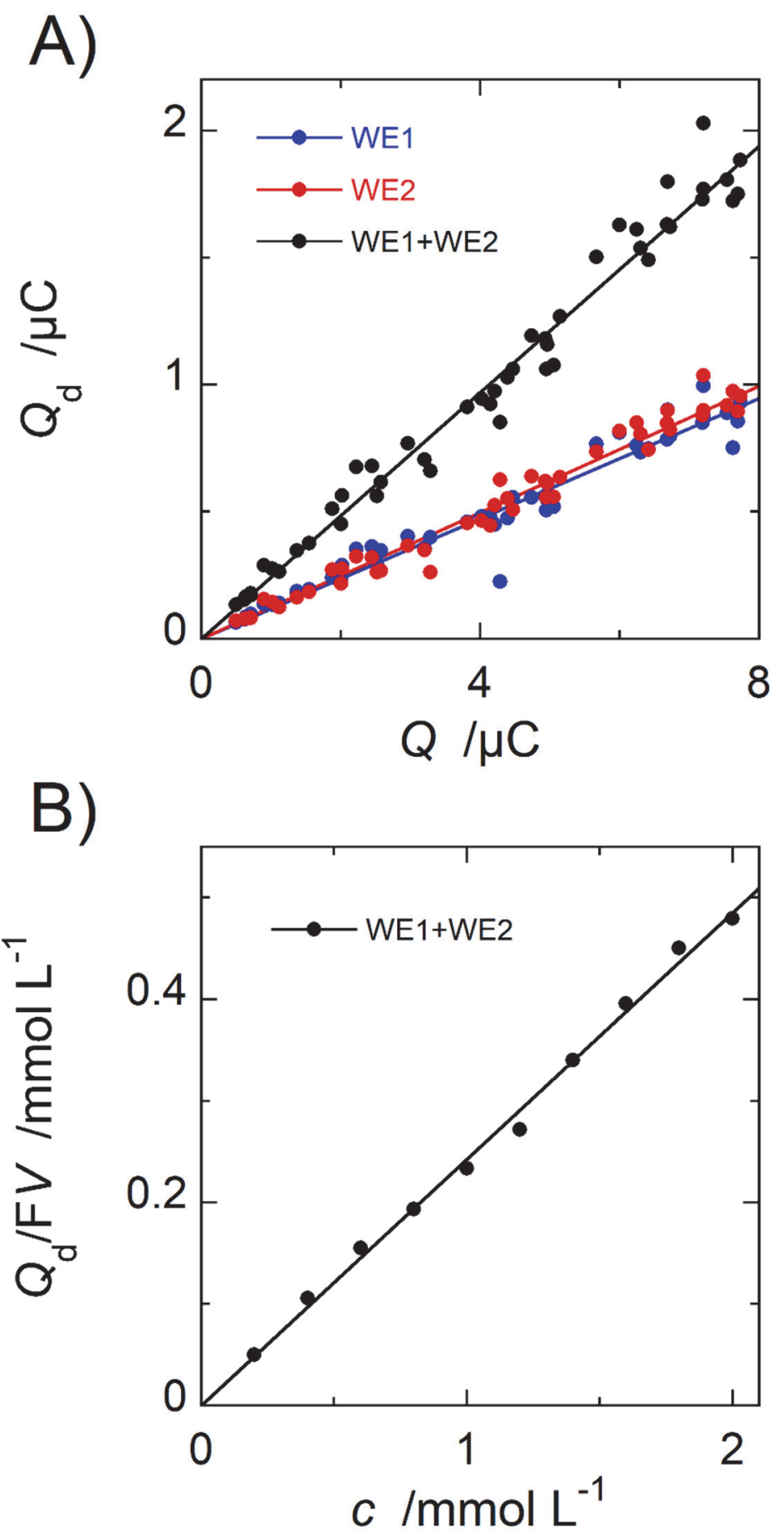

Figure 4: Charges measured during the detection of homogeneous droplets in configuration 4E. A) Comparison between detected charge $Q_{\mathrm{d}}$ and total charge $Q$ of droplet. B) Comparison between concentration deduced from detected charge $Q_{\mathrm{d}}$ and droplet concentration $c$. In A-B) $v=(512 \pm 43) \mu \mathrm{m} \mathrm{s}^{-1}, V=(38 \pm 7) \mathrm{nL}$. Slopes of linear regression are reported in Table 2. 


\section{Heterogeneous droplets - Configuration 5E}

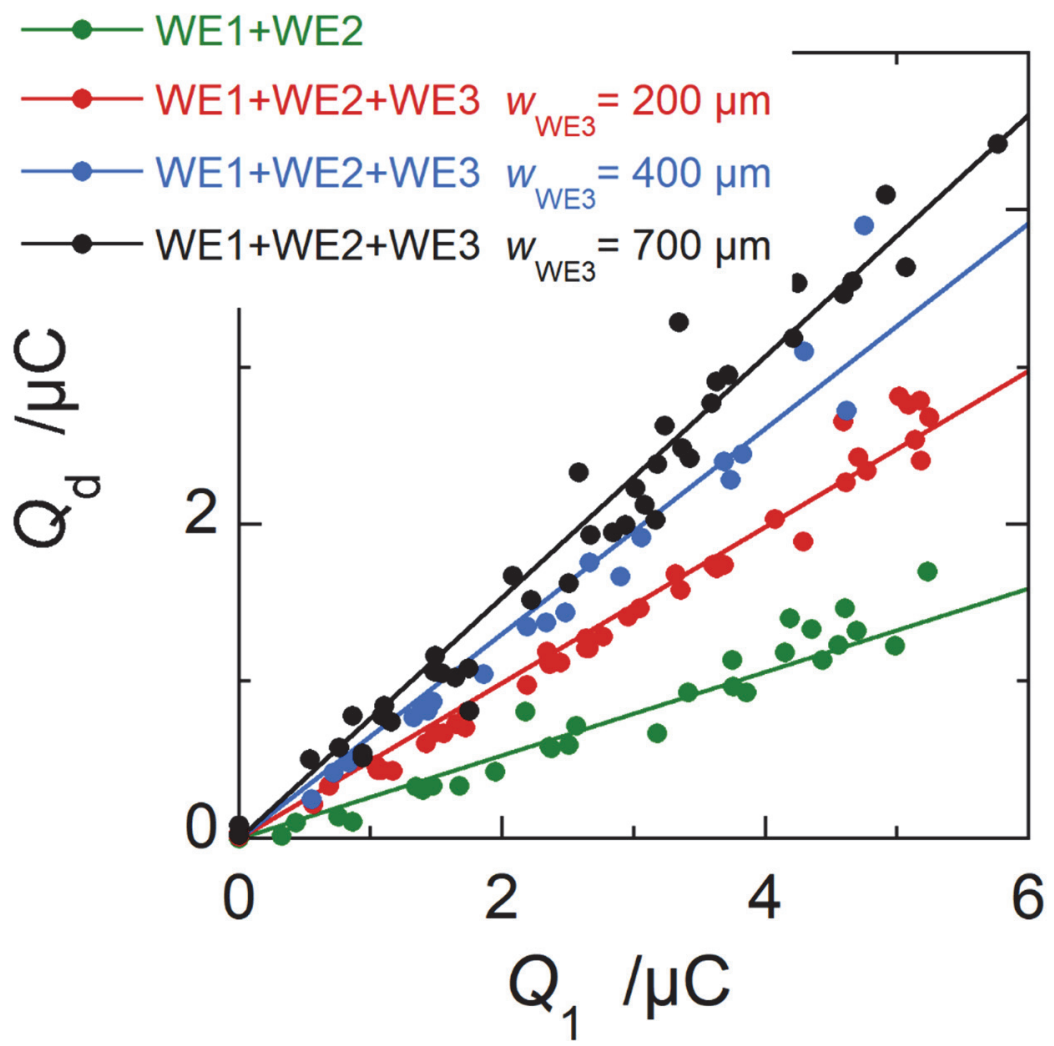

Figure 5: Influence of the electrode size on the detected charge $Q_{\mathrm{d}}$ for heterogeneous droplets in configuration 5E. $Q_{1}$ is the total charge of droplet. In all cases $w_{\mathrm{WE} 1}=w_{\mathrm{WE} 2}=50 \mu \mathrm{m}$. $v=(446 \pm 77) \mu \mathrm{m} \mathrm{s}^{-1}, V=(34 \pm 6) \mathrm{nL}$. Slopes of linear regression are reported in Table 2. 

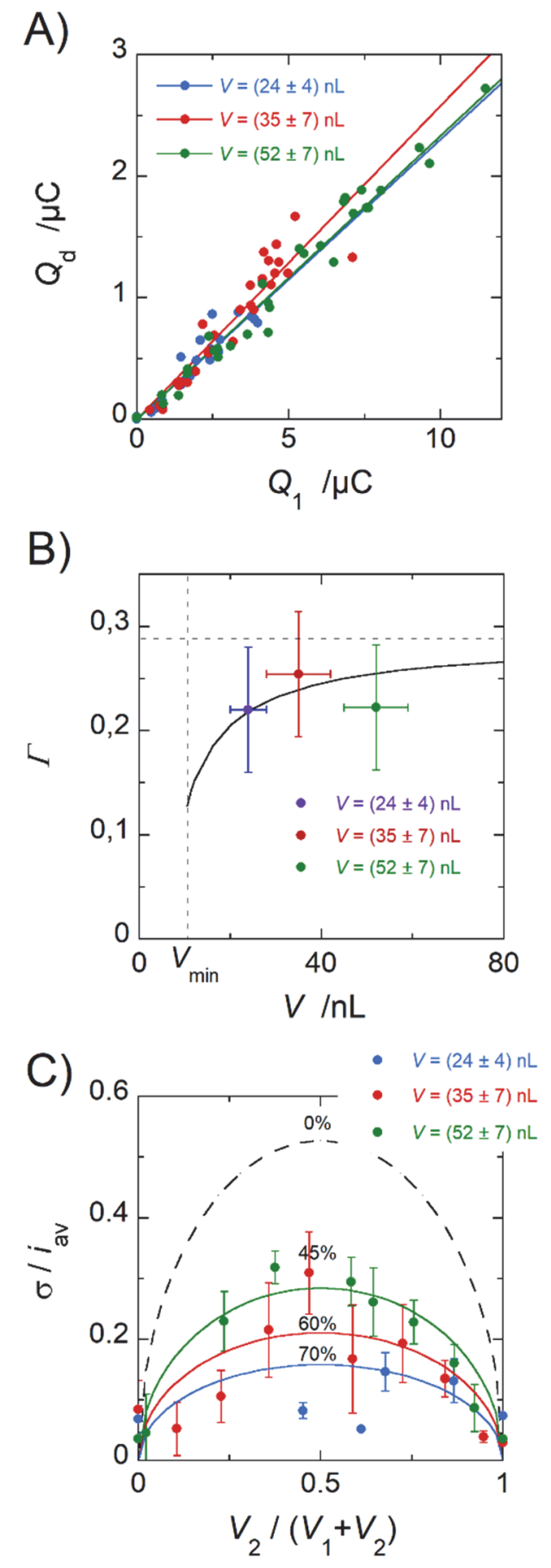

Figure 6: Influence of droplet volumes $V$ on detected charges for heterogeneous droplets in configuration 4E. A) Comparison between detected charge $Q_{\mathrm{d}}$ and total charge $Q_{1}$ of droplet. Values of linear regression slopes are given in Table 2. B) Comparison between theoretical (curve) and experimental (dots) detection rates as a function of droplet volume $V=V_{1}+V_{2}$. The dotted line corresponds to the limiting value achieved when $V$ tends to infinity. C) Evaluation of droplet heterogeneity $\sigma / i_{\text {av }}$ as a function of volume ratio $V_{2} /\left(V_{1}+V_{2}\right)$. The dotted and solid lines correspond to theoretical curves with different mixing efficiencies as indicated on the figure. In (A-C), $V=V_{1}+V_{2} ; v=(535 \pm 99) \mu \mathrm{m} \mathrm{s}^{-1}$. 


\section{A) Heterogeneous droplets - Configuration 5E}

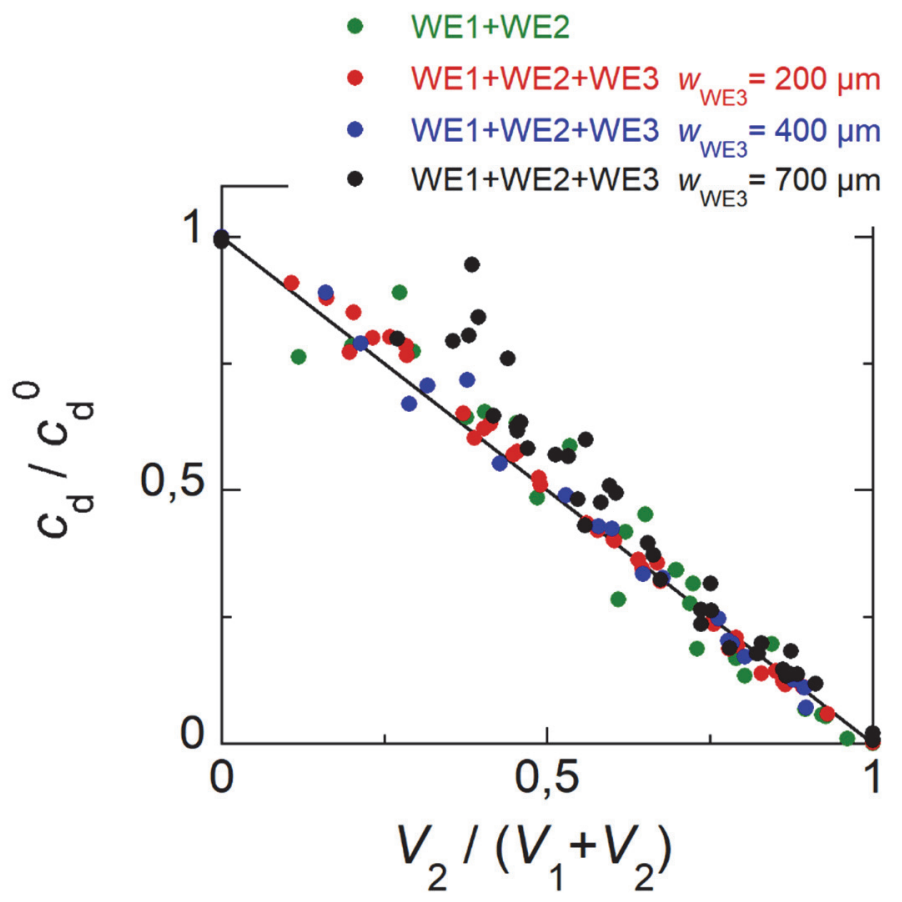

B) Heterogeneous droplets - Configuration 4E

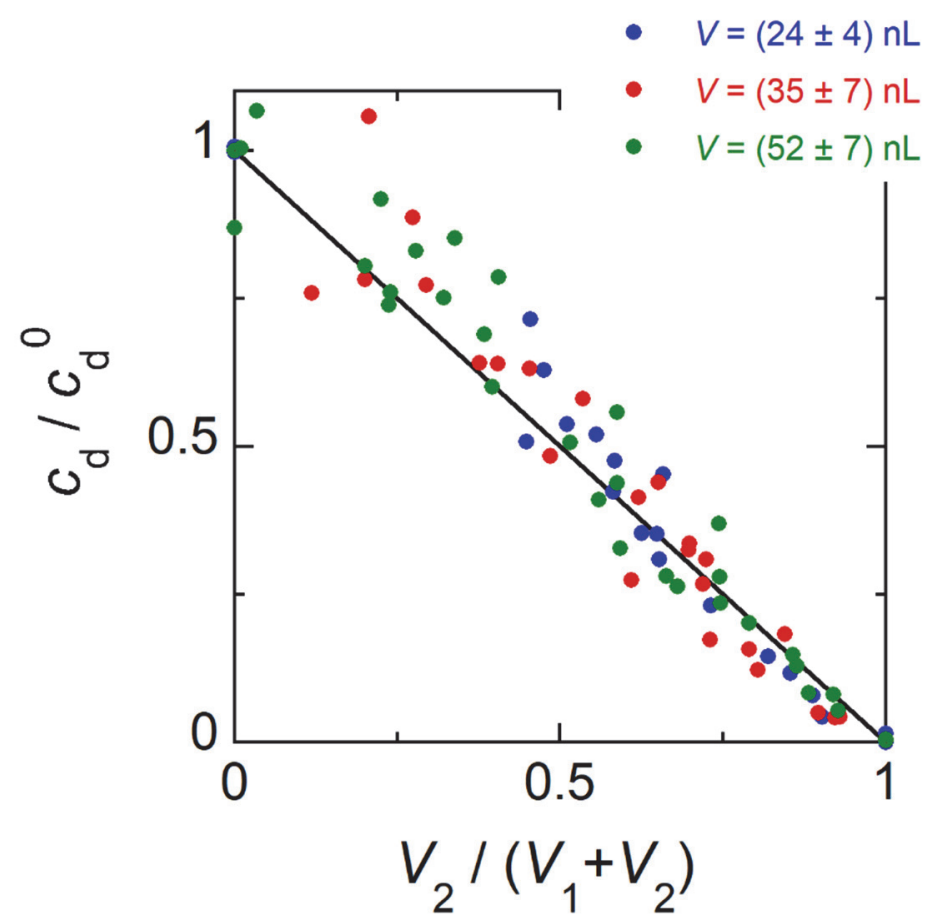

Figure 7: Dilution curves established with heterogeneous droplets after active merging. A) Curves established in configuration 5E from data in Figure 5. B) Curves established in configuration 4E from data in Figure 6A. In all cases $w_{\mathrm{WE} 1}=w_{\mathrm{WE} 2}=50 \mu \mathrm{m} . \quad V=V_{1}+V_{2}$. 


\section{Heterogeneous droplets - Configuration 4E}

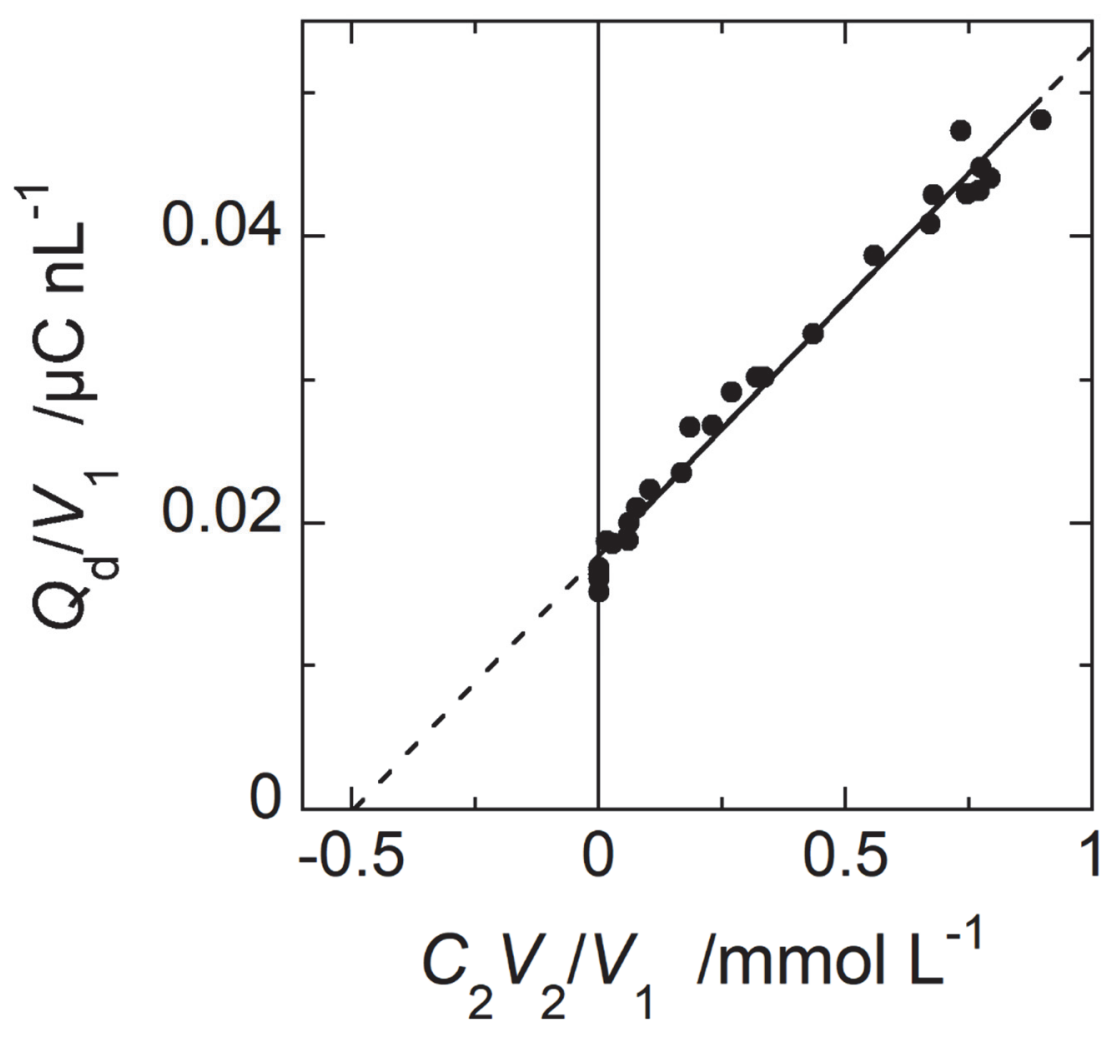

Figure 8: Titration performed after active merging with heterogeneous droplets in configuration 4E. $v=(358 \pm 14) \mu \mathrm{m} \mathrm{s}^{-1}, \quad V=V_{1}+V_{2}=(29 \pm 2) \mathrm{nL}, \quad c_{1}=510^{-4} \mathrm{~mol} \mathrm{~L}^{-1}, \quad c_{2}=9.810^{-4} \mathrm{~mol} \mathrm{~L}^{-1}$. Linear regression $Y=0.017689+0.035631 X, R=0.992$. 
GRAPHICAL ABSTRACT

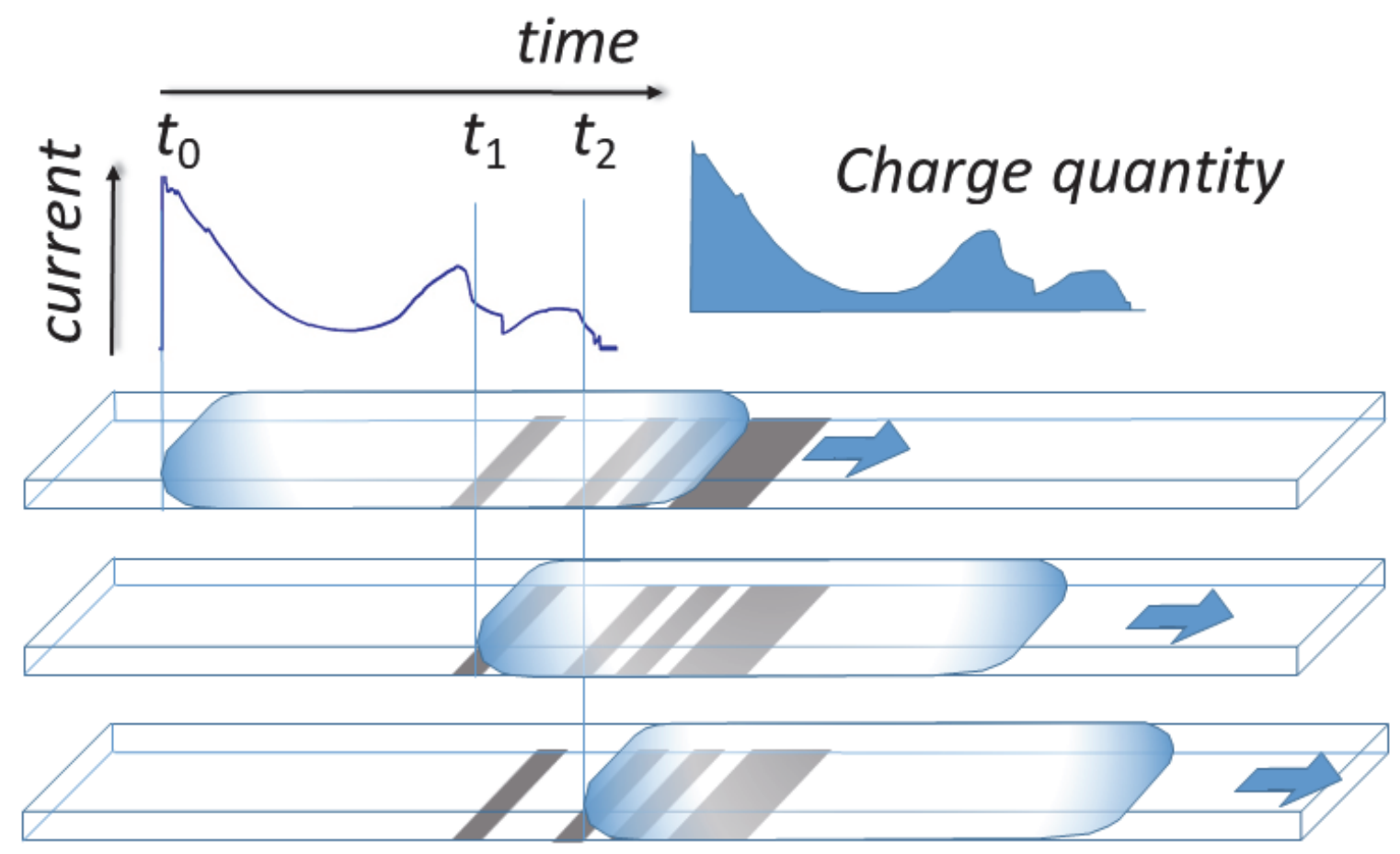

